Fecha de recepción: diciembre 2018

Fecha de aceptación: marzo 2019

Versión final: abril 2019

\section{El Deutscher Werkbund - Peter Behrens. Los Pasajes Del Lenguaje}

Sergio David Rybak*

Resumen: El cambio del siglo XIX al XX, fue testigo de un pasaje violento de los lenguajes en el diseño, la arquitectura y la producción de objetos. En el imperio prusiano se desarrolló la actividad de una institución que junto con sus integrantes fue capital para los nuevos lenguajes y las nuevas actitudes frente a la producción industrializada: el Deutscher Werkbund. Esto se desarrolló con el acompañamiento de empresas como la AEG y por profesionales como Herman Muthesius y Peter Behrens.

Palabras clave: Diseño - institución - lenguajes - morfología - historia - industrialización - arte.

[Resúmenes en inglés y portugués en la página 60]

${ }^{(*)}$ Arquitecto (FAU UBA). Doctor en el área de Historia del Diseño (FADU UBA). Profesor Consulto. Docente en la Maestría de Historia de la Arquitectura, el diseño y el Urbanismo y del Doctorado (FADU-UBA). Especialización en enseñanza universitaria, Carrera Docente (FADU-UBA). Autor del libro El diseño industrial y los productos industriales en la Argentina desde 1952 hasta 1983. Tensiones entre diseño, tecnología y políticas económicas.

\title{
Introducción
}

Kenneth Frampton (1983, 111-112) relata cómo Gottfried Semper al visitar la exposición Universal de Londres da cuenta en 1852 del "impacto de la industrialización y el consumo masivo sobre todo el campo de las artes aplicadas y la arquitectura". La unificación de Alemania bajo el liderazgo prusiano determinó que "una mejora en el diseño, tanto en la artes como en la industria, era esencial para la prosperidad futura”, ya que la única manera que tenía este país de competir en el mercado mundial era sólo mediante el desarrollo de productos de una "calidad excepcionalmente alta".

Es por ello que el Deutscher Werkbund (DWB) no fue un movimiento artístico, ni una tendencia o movimiento en arquitectura, y no fue tampoco una organización de arte con 
una propuesta vanguardista. Fue una institución como parte del proyecto del Imperio prusiano que permitió un giro importante en los inicios del siglo XX en la apreciación y consideración del lenguaje del diseño.

En el Deutscher Werkbund se intentó reunir la mayor cantidad de personas intervinientes en los diversos aspectos de la producción, pero fundamentalmente a artistas, artesanos, industriales y comerciantes. Personas e instituciones que provenían de distintas ciudades del Impero prusiano como así también del Imperio austrohúngaro.

Como lenguajes formales a finales del siglo XIX se utilizaban fundamentalmente dos: los historicismos y distintas líneas del Art Nouveau. Tengamos en cuenta que los lenguajes y las formas son esclavos de su tiempo y tienen para cada momento diversas maneras de manifestarse.

Pretender que esta institución, a través de sus integrantes, tuviera un lenguaje unívoco, algo que se pudiera llamarse lenguaje o estilo Deutscher Werkbund, es errado.

¿Qué pretendía el Deutsche Werkbund o el Imperio prusiano a través del DWB?

Con la guerra franco - prusiana comienza prematuramente la intención de Alemania de estar presente en el nuevo reparto de poder que se desarrollará desde esta guerra hasta desembocar en el estallido de la Primera Guerra Mundial.

Prusia llegó al cambio del siglo, quizás, siendo el país europeo con mayor desarrollo tecnológico aplicado a su industria y, posiblemente, uno de los tres más industrializados del continente. Pero no podía penetrar el mercado inglés.

Para esto, en 1896 la Cámara de Comercio Prusiana envió a Londres al arquitecto Hermann Muthesius como parte del cuerpo diplomático alemán para estudiar en el arte y la producción inglesa las razones de su afirmación en el mercado del cual Alemania no podía desplazarla, y la posibilidad de trasplantar hacia este país algunas de las experiencias.

Muthesius al volver en 1903, desarrolló por un lado sus trabajos particulares y por el otro continuó como asesor del gobierno prusiano. Como parte de ello tuvo la tarea de organizar la reforma del Programa Nacional de Educación en Artes Aplicadas (Gay, Samar 2007).

En 1907, como consejero privado en el Ministerio de Comercio de Berlín, funda con Peter Behrens entre otros, el DWB, aun activo hoy. En ese año Muthesius, según Peter Bruckmann,

... "lanzó una seria advertencia acerca de la superficialidad con que la artesanía y la industria alemana se preocupaban por dotar a sus productos de una, así denominada, forma de "estilo". Lo dijo en una intervención mantenida en la nueva Escuela Superior de Comercio de Berlín, y en tanto aquéllas, artesanía e industria, atañían al ámbito de las artes aplicadas o industriales" (Bruckmann [1932] 1995:75).

Posiblemente a raíz de su idea, doce personas y doce instituciones componían el contingente inicial del llamado a la formación del Deutscher Werkbund.

En la convocatoria estaban Peter Behrens, Theodor Fischer, Josef Hoffman, Wilhelm Kreis, Adalbert Niemeyer, Max Lauger, Josef Olbrich, Bruno Paul, Richard Rimerschmid, J. J. Scharvogel, Paul Schultz-Naumburg y Fritz Schuhmacher (García Roig, 1995). (Frampton, 1983) 
Dentro de las instituciones había asociaciones de artesanos y artistas, fabricantes de cubiertos y accesorios de plata, fabricantes de muebles, editoriales, escuelas de arte, tejedurías, talleres metalúrgicos, talleres de artículos para el hogar y, empresas y organizaciones que estaban comprometidas con la excelencia de la producción artesanal, que consideraban fundamental la idea de encontrar la vía para lograr unir la producción industrial con el arte y las artesanías.

Si se analiza los nombres de las personas que fundaron el Werkbund se puede observar a arquitectos y artistas, mucho de los cuales provenían de una formación académica que estaban o fueron migrando, en mayor o menor medida, hacia distintas formas de Art Nouveau, y desde éste hacia formas más abstractas y simples que dieron lugar a las morfologías aceptadas de la modernidad. A pesar de esto, el clasicismo historicista y las consignas académicas estarán superpuestos a las nuevas propuestas formales y muchas veces, algunos de los integrantes oscilarán entre las diversas propuestas morfológicas.

En Muthesius también se puede ver con respecto a los lenguajes que utilizó desde su formación académica, pasajes y modificaciones de sus propuestas. En Inglaterra adhirió al movimiento Arts \& Craft. En 1904 publicó el libro La casa inglesa que es un análisis, "como un informe" según Torrent y Marín (2007), que constituyó el primer referente "para la reflexión conjunta de los arquitectos, artistas, artesanos e industriales alemanes" (Torrent Marín, 2007:146). Se convirtió a partir de ese momento en un promotor importante de los nuevos enfoques de la producción industrial. Unos meses antes de la apertura del Werkbund, Muthesius dictó una conferencia en la cual se manifestó en contra de los excesos del eclecticismo historicista de la producción alemana, el gusto de la burguesía por el lujo, los ornamentos y los formalismos, inclusive el Jugentstil. Abogó por los valores artísticos, culturales y económicos del "arte industrial". Con esto logró importantes adhesiones en el campo artístico y empresarial.

Por otra parte, ya en la tercera exposición de Artes Aplicadas de Dresde de 1906 se pudo apreciar que el Jugentstil perdía importancia (Torrent Marín 2007; Bröhan Berg 1994) como también el crecimiento de un estilo más severo.

El lenguaje de las formas había sido alterado girando hacia una propuesta de expresión más rigurosa que enfatizaba la función del objeto. El objetivo de esta exposición fue la promoción de nuevos estándares de diseño en los campos de la arquitectura, artesanías e industrias para conformar una nueva estética. La innovadora experiencia de la exposición de Dresde estableció los estándares para el Deutscher Werkbund que se fundó el año siguiente.

Allí vemos la utilización del término "funcional". En un principio el término "funcional" se equiparaba a lo orgánicamente vegetal, según la máxima de Luis Sullivan (Heskett 2002: 36; Burkhardt Fuchs, 1988). Luego se abandonan los conceptos y las formas que llevan a lo orgánicamente vegetal, limitándose a elementos objetivos concebidos a través del viraje a formas rectas y con figuras y volúmenes geométricos reconocibles por su regularidad. Antes de la apertura del DWB en las asociaciones de empresarios, artistas y escuelas de arte se discutía también acerca de sus objetivos económicos, de las características de los productos y las funciones de cada uno. Se manifestaron dos posiciones: una conservadora que defendía las formas de producción y morfologías existentes y la que promovía el cam- 
bio hacia una nueva estética industrial como una expresión acorde con las características que sustentaban los avances tecnológicos de la industria y sus posibilidades.

Por otra parte, Burkhardt y Fuchs (1985) mencionan que Muthesius, en su discurso de aceptación de la primera Cátedra de Artes Aplicadas en la Escuela Comercial Superior de Berlín, vertió demandas acerca de los lenguajes y características nuevas en la producción a los artistas, industriales y fabricantes, que posteriormente pasaron a formar parte de los objetivos y estatutos del DWB.

Hay que aclarar que los temas que debía afrontar el Werkbund Alemán eran los siguientes: El morfológico, es decir, cuál era el mejor lenguaje para que el público aceptara los productos alemanes. De este se desprendió otro muy importante en el Werkbund, la educación del público.

Otra cuestión fue la mala calidad de lo producido en Alemania, que llevó a estudios para elevar los estándares de producción con el objetivo que los productos alemanes sean conocidos como los "mejores del mundo".

Otro punto que importó era cómo se integraban los lenguajes, la tecnología y la producción industrial, razón por la cual se realizó el llamado a integrar el DWB a artistas, artesanos, arquitectos, industriales, cámaras de comerciantes y escuelas de arte y de artesanías. Los estatutos iniciales del DWB aclaraban: "El objetivo de esta asociación es el ennoblecimiento del trabajo industrial en la colaboración del arte, industria y artesanía a través de educación, propaganda y postura unificada ante asuntos relacionados con ellos". (Citado por Burkhardt Fuchs 1985:37).

La postura unificada fue una meta difícil de cumplir. Muthesius pregonaba una forma de trabajo sin arte ${ }^{1}$. Ante esto algunas personalidades que provenían de grupos de artistas, sobre todo del Jugentstil, que basaban la concepción de la forma en la idea del artista creador, se oponían al concepto que abrazaba Muthesius. En esta posición se ubicaba particularmente Van de Velde.

Muthesius le reprochó a la industria que era casi inmoral producir cada mes novedades “originales" y "baratas". Según Burkhardt, Muthesius demandaba la fabricación de cosas durables en cuanto al material y al diseño, expresando que era necesario desechar el individualismo en el diseño para crear algo de validez universal: los tipos.

Este tema volvía repetidamente a las discusiones en el DWB. En 1914 en la exposición del DWB que se realizó en Colonia, los voceros de las dos posiciones, Henry van de Velde y Hermann Muthesius, se enfrentaron por las premisas que se deberían seguir.

Van de Velde, defendió su posición expresando que mientras exista la inspiración del artista no podrá ser posible la tipificación dejando entrever que esta le restaría riqueza a la creación. Muthesius por el contrario era partidario de la tipificación y estandarización industrial. (Burdek, 1994, 24) En esa votación salió elegida la posición de Van de Velde.

La Primera Guerra Mundial puso fin a esta discusión sobre el vínculo entre arte e industria forzando la toma de decisiones para una producción más racional y económica. Durante la guerra, además, se intensificaron los conceptos de "lo nacional" inherente al Werkbund desde el principio. También a caballo de la guerra se crearon las Normas de Industria Alemanas (DIN en su sigla alemana). 
No hay que olvidar que en el Werkbund no se produjeron objetos, sí gráficas de publicidad de eventos propios y el diseño y construcción de edificios de las exposiciones a las que concurría.

\section{AEG y Peter Behrens}

La AEG (Allgemeine Elektricitäts-Gesellschaft) fue creada en 1887 partiendo de una fábrica anterior de bombitas eléctricas. El ingeniero industrial Emil Ratenau obtuvo el derecho de explotar en Alemania la patente de Edison a partir de la cual creó una compañía. Esa fue la base de la AEG.

Esta empresa, que basó su accionar en los conceptos de una producción eficaz, diversificó sus artículos y comenzó también a producir motores y dínamos junto a electrodomésticos y una serie de productos ligados a las nuevas tecnologías industriales.

Se debe considerar que con el uso de la electricidad aparecieron una cantidad de productos nuevos sin antecedentes históricos, a partir de los cuales se podían crear visiones estéticas alejadas de los repertorios formales existentes. La AEG fabricaba un vasto surtido de productos en donde se podían ver el desarrollo de los nuevos conceptos.

Después de la fundación del DWB, la AEG adhirió a los principios de Muthesius.

En la empresa se intentó producir todo bajo una imagen global para reforzar una imagen identitaria unitaria de la empresa, lo que ahora se conoce como identidad corporativa.

Peter Behrens, como se mencionó, uno de los participantes importantes del DWB, construyó en 1908 la fábrica de turbinas de la AEG que se tomará como estudio de caso para desarrollar un modelo de análisis. También se analizará un objeto de la misma empresa para la que trabajó desde 1907. Según algunos teóricos del diseño e historiadores, la creación del DWB y la contratación de Behrens por la AEG, no están conectadas, salvo por estar en el mismo país en donde se desarrollaban intensas discusiones acerca de cómo se debía producir el mejor "producto alemán".

Peter Behrens nació en Hamburgo en abril de 1868 y comenzó sus estudios de pintura en 1886 en la Academia de Arte de Karlsruhe. Su educación artística inició en el área de la pintura. Fue uno de los miembros fundadores de la Secesión Muniquesa en 1883. Hacia el fin de la década de 1890 abandonó la pintura y orientó su actividad hacia las artes aplicadas (Buddensieg 1979).

En 1899 se afincó en la colonia artística de Darmstaadt en donde fue una de las más importantes voces junto a Josef María Olbrich. En enero de 1903, año en que fue fundado el Wiener Werkstätte y que retornó Muthesius de Inglaterra, fue nombrado director de la Academia de Artes y Oficios de Dusseldorf.

Se puede apreciar en esta época a partir lo producido en Darmstaadt, un corrimiento desde el Jugendstil hacia una geometría más abstracta.

En 1907 los directivos de la AEG convocaron a Behrens para desempeñarse como diseñador gráfico. Tilmann Buddensieg (1979) comentó que desde ese momento Behrens manifestó una marcada influencia por el Clasicismo Prusiano. Torrent y Marín (2007) 
expresan: "Su obra cumbre en esta línea de actuación fue la fábrica de turbinas de la compañía en Berlín (Imagen 1) edificio concebido a la vez, con ánimo industrial y aspiraciones clásicas" (Torrent, Marín 2007:156). Hay que destacar que su pasado modernista de pertenencia al Jugendstil, fue modificado por las demandas de sus contratantes. En este caso se adentró paulatinamente hacia una visión total del diseño.

\section{Fábrica de Turbinas}

El ánimo industrial de Peter Behrens está manifestado en las grandes superficies vidriadas para el aprovechamiento de la luz natural, tanto en la parte frontal como en la lateral y en la nave con estructura de hierro sin apoyos intermedios. Pero no termina de definir la fachada lateral como una piel que envuelve una nave industrial.

Torrent y Marín (2007), plantean la clasicidad en la visión de la nave ya que fue concebida, según ellos, como un monumento. Parecida conclusión es la que arriba Pevsner $(1977,222)$ cuando lo define sin titubear "el edificio industrial más hermoso de todos los construidos hasta esa fecha", agregando que "el resultado es una perfecta obra de arte, tan perfectamente equilibrada que sus enormes dimensiones no se captan, a menos que se compare con las personas de la calle".

Se puede considerar su clasicidad en la concepción tectónica, la clara diferenciación entre lo soportado y lo soportante, aunque no se observa un frontis o un tímpano clásico. Su fachada esta rematada por un techo articulado en donde no se observa la estructura de hierro, ya que está oculta por el tímpano. Los volúmenes de las esquinas se leen perfectamente como volúmenes sustentantes. La estructura sustentante real del edificio, es de hierro lo mismo que las cabreadas articuladas que sostienen el techo.

En el lateral vidriado se marcan los tramos que dan lugar a los pies derechos que sostienen las cabreadas, configurando una estructura ordenada y modulada lo cual era una manifestación de la racionalidad de la obra.

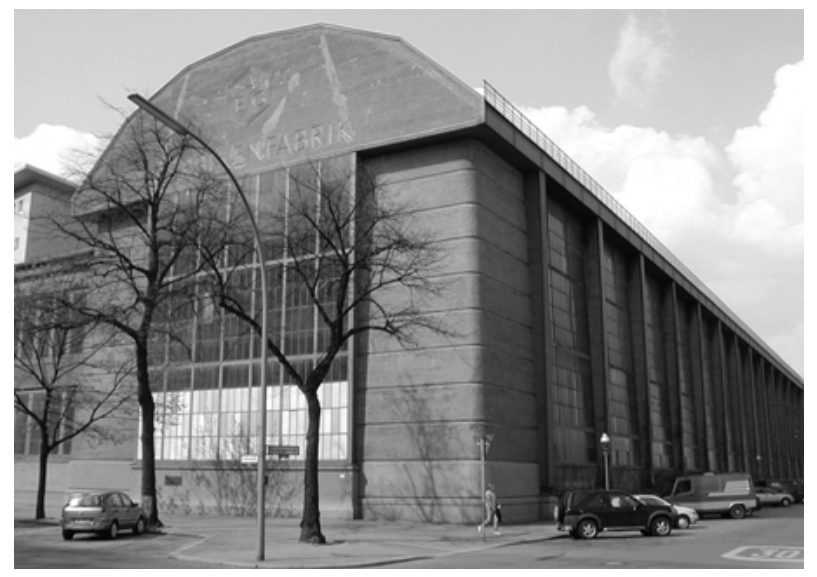

Imagen 1. Fábrica de turbinas de la AEG, 1908. Peter Behrens. Fuente: https:// europeancollections.wordpress. com/2018/08/17/peterbehrens-150/aeg/ 
La imagen nos muestra que el edificio fue pensado para destacar esta visión perspectívica de la misma manera que en los tempos clásicos griegos. En la nave se puede observar, cómo se mencionó, un volumen prismático con cuatro importantes volúmenes macizos en las esquinas y que el volumen del techo está apoyado en estas cuatro esquinas. Los soportes laterales de la estructura remiten al ritmo de las columnas laterales de los templos griegos. La ley que rige a esta nave industrial surge a partir de considerar un eje longitudinal que se conceptualizará también como un plano de simetría. La fachada se regirá por este plano, dejando de lado una parte lateral de la construcción, como si estuviese adosada. Considerando esto, en la fachada se puede observar el isologo de la fábrica que se diseñó con figuras hexagonales, que para Torrent y Marín nos remitirían a la laboriosidad de las abejas. Por debajo del isologo colocó la función del edificio "Fábrica de turbinas" (Turbinenfafrik). Respetando la simetría, el isologo y el texto fueron colocados en el centro del "tímpano".

Desde el exterior el volumen del techo se puede percibir como compacto. En cambio, el prisma inferior se podría apreciar con la intención de componer los llenos y vacíos, salientes y entrantes, avances y retrocesos de la superficie exterior.

En los volúmenes de las esquinas, Behrens adoptó la forma tectónica que desarrolló en la concepción del edificio. Las reglas académicas dictaminaban que en los edificios de varios niveles, el primero debía expresar que era el que más peso soportaba con sus materiales y formas. Esta expresión del soporte mayor en el primer nivel debía alivianarse en los niveles superiores.

Behrens logró esto en un único nivel (nivel +/- 0,00), modificando la verticalidad del paramento en los volúmenes de las esquinas que algunos escritores remiten a los pílonos egipcios, más anchos abajo y afinándolos a medida que se levantan.

Esto no sucedió con los paramentos de vidrio del frente que son verticales al ras del tímpano que sobresale del muro inclinado de la esquina, situación que se invierte en el paramento lateral. Allí la parte vidriada está inclinada y, lo que es el soporte del techo, es vertical y de hierro. Hay que considerar también que la partición del frente vidriado respetó también los conceptos de la simetría con el que fue concebido el edificio.

Además, mientras los frentes de vidrio no manifestaban texturas, los volúmenes de las esquinas se expresaron como si fuesen realizados con enormes piedras, sumado a que las características del material oponen lo liso y lo texturado de los vidrios y el revoque. A partir de esta oposición se puede plantear el par comparativo de lo vacío y lo lleno o lo translucido y lo opaco.

También puede observarse que el edificio no fue tratado como si tuviese una piel envolvente, característica de las construcciones industriales que se habían comenzado a construir, sino que opera desde las tensiones que produce el juego de opuestos: lo liviano y lo pesado, lo vacío y lo lleno, lo transparente y lo opaco.

Si bien la imagen total es de tectonicidad hay resoluciones formales que contradicen esta propuesta ya que se pueden observar detalles que podrían ponerla en crisis, es decir que no hay una ley general que pueda reforzar la idea propuesta inicialmente. 


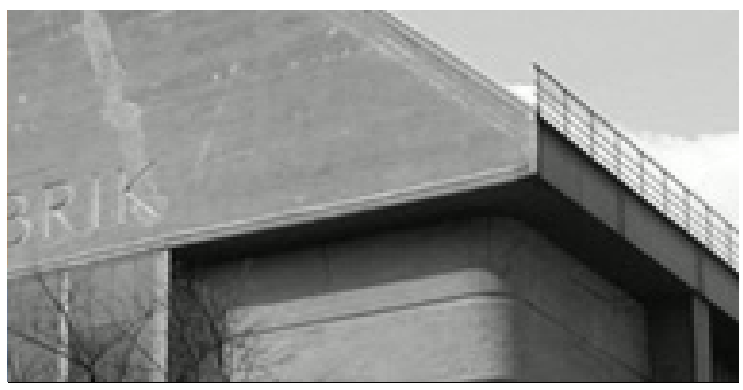

Imagen 2. Fábrica de turbinas de la AEG, 1908. Peter Behrens. Detalle del ángulo del edificio. Fuente https://europeancollections. wordpress.com/2018/08/17/peterbehrens-150/aeg/

Imagen 3: Interior Fábrica de Turbinas AEG, c. 1920.

Fuente: Centro Alemán de Documentación para la Historia del Arte Bildarchiv Foto Marburg

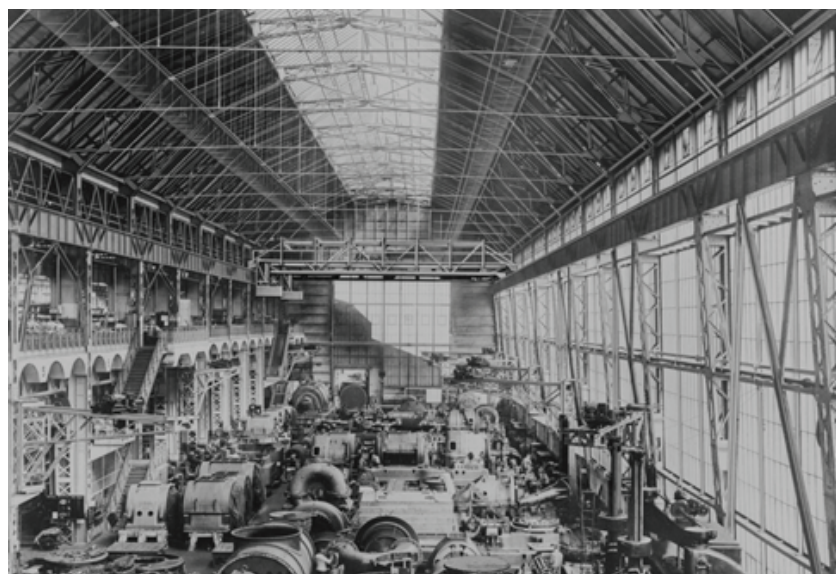

A modo de ejemplo: en la esquina superior del edificio (Imagen 2) se puede observar que el tímpano de mampostería tiene un borde de chapa que es coincidente con el material del techo cooincidente con el cenefón lateral que le da terminación a la estructura de hierro y el desagüe pluvial de la cubierta. El cuestionamiento al orden tectónico aparece en la esquina maciza que se retira hacia adentro permitiendo que en el frente el plano vidriado sea el protagónico.

Para Leonardo Benévolo $(1978,422)$ "el tono general es sobrio y macizo" y los elementos funcionales se encuentran ordenados con una solemnidad wagneriana.

Sin embargo, el interior de la fábrica permite observar el valor que Behrens le otorga a la luz natural y a la espacialidad, a diferencia de los sórdidos talleres industriales de la época. El espacio interior no expresa la aparente densidad exterior, sino que determina un ámbito fluido y liviano.

Para Boztepe $(2012,39)$ esta amplitud permite ordenar de manera fordista los procesos industriales: "El diseño claro, la facilidad de intercambio y el movimiento de productos hacia adelante, y la movilidad sin trabas de herramientas, máquinas o camiones requieren pasillos abiertos, despejados y bien iluminados..." 
Este juego de opuestos y esta intencionada ambigüedad entre lo meramente funcional y lo pretendidamente estético, determinó que "lejos de ser un diseño contundente en hierro y vidrio (como el cobertizo ferroviario del siglo XIX) la Fábrica de Turbinas de Behrens era una consciente obra de arte". (Frampton, 1983, 113) Esta dualidad también se manifiesta en la contraposición del edificio contenedor ordenado y su contenido un tanto aleatorio y desordenado, donde lo primero se impone sobre lo segundo.

En el mismo sentido, Manfredo Tafuri y Francesco Dal Co $(1982,86)$ expresan cómo Behrens plantea una esencialidad tecnológica "que llega hasta la brutal denuncia de elementos estructurales, como los grandes pernos de hierro o la contraposición de las superficies vidriadas desnudas con las pilastras portantes firmemente molduradas."

Habiendo analizado este edificio, se analizará un objeto realizado por la fábrica AEG y diseñado por Behrens.

Es muy probable que los pares conceptuales de los análisis de obras de arquitectura, no sean pertinentes al analizar productos, en general mucho más pequeños y con otras características de proyecto y con otras necesidades tecnológicas de fabricación.

Como ya mencionamos, Peter Behrens había adscripto al Art Nouveau, pasando del orgánico al geométrico. Desde allí no podía separarse de la visión individualista de la unión de los artesanos y artistas. Esto implicaba la consideración de las obras como creación individual, tanto desde la concepción como en su sistema de producción. También en cuanto a la concepción de la importancia del ornamento. Hasta Adolf Loos no se criticó al ornamento en sí, sino el lenguaje formal utilizado, sea historicista u otro nuevo.

En el continuo pasaje en los lenguajes y concepciones que aplicará Behrens está su riqueza. En el sistema de producción de los objetos diseñados por Peter Behrens en la AEG está presente su concepción muy cercana a la de Muthesius.

\section{Lámpara Eléctrica}

La lámpara colgante que realizó entre 1908 y 1909 (Imagen 4) muestra una nueva forma de pensar el producto: La lámpara colgante está concebida en partes.

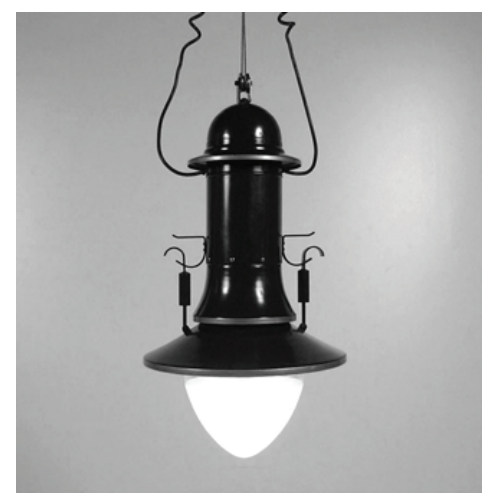

Imagen 4. Lámpara colgante AEG. 1908-1909. Peter Behrens. Fuente: https://sdtb.de/fileadmin/user_upload/_ tem/01_Das_Museum/02_Historisches_Archiv/AEGHistorie_dtsch_DS.pdf 
Las tres partes metálicas de chapa esmaltada negra fueron pensadas para un armado simple, en secuencia y que no tenga curvas y contracurvas. Fue producida con chapa repujada por medios mecánicos. Los sistemas de traba entre cada parte también son simples con un desarme sencillo. El artefacto se sostiene simplemente de una cuerda. Los cables conductores de electricidad entran por una hendidura entre el primer y segundo tramo de chapa logrando una contrapendiente para detener el paso del agua de lluvia. La pieza del primer tramo (superior), en un ánimo de marcar la unión o ensamble, termina en una virola dorada de bronce que resalta contra el cuerpo negro brilloso de la lámpara. En el tercer cuerpo (inferior) se fija la opalina traslúcida. Tanto el segundo tramo (medio) como el tercero (inferior) llevan como terminación una virola dorada de bronce. Entre la chapa inferior y la media es muy perceptible el mecanismo diseñado para accionar la separación entre las dos. Las virolas indican el fin de cada tramo y en donde están los encastres y la posibilidad del desarmado de cada sector de la lámpara.

Boztepe (2012. 59) señala que "una forma agradable es una forma basada en un orden geométrico" ya que Peter Behrens afirmaba en 1909 que la simplificación armónica que buscaba se sustentaba en "proporciones claras" y no en una "ornamentación rica"

El cambio de tecnología de iluminación a gas por eléctrica a fin del siglo XIX posibilitó que la tipología de los artefactos que utilizaban lámparas eléctricas pudiese funcionar en cualquier posición, también hacia abajo. Con la iluminación a gas o a velas, hubiese sido un sinsentido fabricar una lámpara colgante cuya fuente de luz estuviese orientada hacia abajo. No hay que olvidar que los artefactos de iluminación eléctricos ya habían sido diseñados y producidos por artistas o fábricas que adscribían al Art Nouveau. Pero para la producción masiva de artefactos se necesitaba una distribución de electricidad en áreas más importantes que las localizadas por generadores individuales. Esto se logró al filo del cambio del siglo y posibilitó la producción en serie de las lámparas y nuevos artefactos para esta tecnología.

No puede existir en una lámpara colgante de chapa el principio de tectonicidad o de relaciones de masas o llenos y vacíos. Sí podemos analizar el de opacidad y transparencia, como también el análisis de texturas pero de manera diferente de los que se podría analizar en un edificio.

No podemos pensar líneas conceptuales de análisis considerando la coherencia entre el edificio tomado como caso y el objeto analizado. Esta coherencia entre distintos campos se dio de manera general en el barroco, quizás el último movimiento que fue coherente entre sus distintas áreas. A modo de ejemplo, sí podríamos analizar objetos y edificios realizados por los integrantes del movimiento moderno.

Resumiendo, entre el edificio y los productos realizados en la AEG no existe posibilidad de homogeneizar los criterios de creación y de análisis ya que pertenecen a universos diferentes.

En el caso de la fábrica de turbinas, persiste la presencia de la clasicidad pero el diseño de la lámpara eléctrica se apartó de las improntas clasicistas, del ornamento y el Art Nouveau. Cada parte está pensada como integrante de una estructura en función del sistema productivo utilizado y de las nuevas morfologías propuestas. 
En cuanto a las terminaciones, los conceptos y pares con los que se opera en el análisis entre las construcciones de edificios y los objetos, están en función de conceptos diferentes. Las texturas y colores también son diferentes debido a los materiales que se usan en la construcción de cada uno.

En la concepción de las formas, se puede analizar como Peter Behrens posibilitó el pasaje de un lenguaje a otro debido a su percepción de las diferentes propuestas que podía realizar. Con la lámpara eléctrica comprendió que debía modificar sus premisas conceptuales y operar sobre la modificación que podía generar en un producto sin historia y con la posibilidad de un código nuevo.

\section{Notas}

1. Como "trabajo sin arte" Muthesius se refería a lo siguiente: Sin la importancia de la subjetividad en la concepción del diseño, tendiente a un grado mayor de objetividad que relacionaba con lo racional. De hecho oponía subjetividad con racionalidad.

2. Esto se puede observar en la perspectiva realizada para el proyecto por Behrens.

3. Desde 1875 comenzaron a construirse instalaciones eléctricas para lámparas de arco con generadores, para abastecer de fluido eléctrico a lugares determinados como la Gard du Nord en Francia, o las que se construyeron en Deptford para la London Elextricity Suply Corporation en 1889. La central eléctrica de Édison en Nueva York entró en funcionamiento en 1882. La primera gran usina hidroeléctrica comenzó a construirse en las cataratas del Niágara en 1886. Una de las primeras fábricas importantes de lámparas de fibra de carbono se creó en 1886 con la asociación de Édison y Swan. Cuando caducó la patente de este tipo de lámpara se separaron. No hay que olvidar que con Édison fue que Ratenau obtuvo el acuerdo para la patente de las lámparas incandescentes que fabricaría con exclusividad en Alemania (Derry, Williams 1977).

\section{Lista de Referencias Bibliográficas}

Benévolo, Leonardo (1978) Historia de la Arquitectura Moderna, Barcelona, Editorial Gustavo Gili.

Boztepe, Uygar (2012) Aeg \& Peter Behrens: Symbolism in The First Corporate Identity Design en http://library.iyte.edu.tr/tezler/master/mimarlik/T001049.pdf Extraído el 12 de febrero de 2019

Bruckmann P. (1995 [1932]) "La fundación de la Deutscher Werkbund 6 octubre 1907" en Cuaderno de notas. Extraído el 26 de enero de 2019 https://www.google.com/search?q $=\mathrm{ACta}+\mathrm{de}+$ fundaci $\% \mathrm{C} 3 \% \mathrm{~B} 3 \mathrm{n}+\mathrm{del}+$ Werkbund \&tbm $=$ isch\&source $=$ univ\&sa $=\mathrm{X} \& v e d=$ 2ahUKEwiszdWg_4vgAhXmHLkGHUXkDcsQsAR6BAgFEAE\&biw=1419\&bih=737 \#imgrc=7B4PNG3ZtF47rM:

Bröhan T., Berg T. (1994) Avantgarde Design. Alemania. Editorial Taschen

Buddensieg T. (1979) Industriekultur. Peter Behrens and the AEG. Berlin. Gebr. Mann Verlag. 
Bürdek, Bernhard (1994) Diseño, Historia, Teoría y Práctica del Diseño Industrial. Barcelona, Editorial Gustavo Gili

Burkhardt F., Fuchs H. (1985) Producto Forma Historia. Stuttgart. Heinrich Fink GmbH+Co. Derry T. K., Williams T. (1977) Historia de la tecnología (II) desde 1750 hasta 1900. España. Siglo XXI.

Gay A, Samar L. (2007) El diseño industrial en la historia. Córdoba. Ediciones TEC

García Roig, J. Manuel (1995) “La Deutscher Werkbund”, en Cuaderno de notas. Extraído el 26 de enero de 2019 https://www.google.com/search?q=ACta+de+fundaci\%C3\%B3 $\mathrm{n}+$ del+Werkbund\&tbm $=$ isch\&source $=$ univ\&sa $=$ X\&ved $=2$ ahUKEwiszdWg_4vgAhX mHLkGHUXkDcsQsAR6BAgFEAE\&biw=1419\&bih=737\#imgrc=7B4PNG3ZtF47rM:

Frampton, Kenneth (1983) Historia crítica de la arquitectura moderna, Barcelona, Editorial Gustavo Gili.

Heskett J. (2007) El diseño en la vida cotidiana. Barcelona. Editorial Gustavo Gili

Pevsner, Nikolaus (1977) Pioneros del Diseño Moderno de Wiliam Morris a Walter Gropius, Buenos Aires: Ediciones Infinito.

Tafuri, Manfredo - Dal Co, Francesco (1982) Historia de la Arquitectura Contemporánea, Tomo 1, Buenos Aires, Viscontea

Torrent R, Marín J. M. (2007) Historia del Diseño Industrial. Madrid. Ediciones Cátedra.

\begin{abstract}
Changes from the nineteenth to the twentieth century witnessed a violent passage of languages in design, architecture and objects. In the Prussian empire, the activity of an institution together with its members developed what was capital for those new languages and new attitudes towards industrialized production: the Deutscher Werkbund. This has been developed with the support of companies such as the AEG and by professionals such as Herman Muthesius and Peter Behrens.
\end{abstract}

Keywords: Design - institution - languages - morphology - history - industrialization - art.

Resumo: A mudança do século XIX para o XX testemunhou uma passagem violenta de linguagens no design, na arquitetura e na produção de objetos. No império prussiano, desenvolveu-se a atividade de uma instituição que, juntamente com seus membros, era a capital para as novas linguagens e novas atitudes em relação à produção industrializada: o Deutscher Werkbund. Este foi desenvolvido com o apoio de empresas como a AEG e por profissionais como Herman Muthesius e Peter Behrens.

Palavras chave: Design - instituição - linguagens - morfologia - história - industrialização - arte.

[Las traducciones de los abstracts fueron supervisadas por el autor de cada artículo] 